\title{
Rundschau
}

Adjuvante Radiotherapie 39

Akute idiopathische Kolondilatation 236

Angiogenese 113, 199

Aspirin 205

Assistierte Reproduktionstechniken 3

Azidoserate, kindliche 76

Chromosomenanalyse 9

Dammschnitt- und Dammrissrate 76

Differentialdiagnose 215

Diskordante Untersuchungsergebnisse 9

Dünnschichtzytologie ThinPrep ${ }^{\circledR} 81$

Eigenfetttransplantation, Labia majora 23

Endometriose, Häufigkeit 118

-, neue Therapieansätze 113

-, operatives Management 124

-, Rezidiv 124

Endozervikale Zellen 81

Fertilität 124

Fetales Wachstum 57

Frühgeburtsrisiko 64

Gastrointestinale Komplikation 236

Gestose 209

- -Frauen, Selbsthilfeorganisation 222

HELLP-Syndrom 215, 222, 236

Heparin 205

Humanes Papillomvirus 88

Humangenetische Beratung 9

Hypertonie 209

Immunologie 199

Infektionsrate(n) 76

- bei Neugeborenen 33

Interferon 39

\section{Kaiserschnitt 70}

Keimbesiedelung des Wassers 33

Klitorisverkleinerung 23

Konservative Therapieansätze 118

Lebererkrankung 215

Liposuktion, Schamhügel 23

Magnetresonanztomographie, Becken 226

Maternal-fetale Maladaptation 199

Mehrlinge 14

Mehrlingsschwangerschaft(en) 3, 9, 57

Mikroangiopathie 215

Münchener Nomenklatur II 81
Neoplasien 226

Nymphektomie 23

Ogilvie-Syndrom 236

Partnerschaft 132

Pathophysiologie 113

Peroxisom-Proliferator-aktivierte Rezeptoren $\gamma 113$

Plazentation 199

Postpartale Komplikation 236

Präeklampsie, Nachbetreuung 222

-, Pathophysiologie 199

-, Prävention 205

-, Therapie 209

Pränatale Diagnostik 9

- Medizin 14

Primäres malignes Melanom der Vagina 39

Psychosomatische Unterstützung 132

Psychosoziale Belastung 132

Radiochemotherapie 88

Retrograde Menstruation 113

Risikoschwangerschaft 70

Schamlippenverkleinerung 23

Schmerz 132

Schulterdystokie 76

Schwangerschaft 236

Sectio caesarea 236

Sentinellymphonodektomie 39

Sexualität 132

Single-Embryo-Transfer 3

Thrombophilie 205

Ultraschall 14

Vagina 226

Vaginale Geburt, Zwillinge 70

Vulva 226

Vulvakarzinom 88

Vulväre intraepitheliale Neoplasie 88

Wachstumsdiskordanz 57

Wächter-Lymphknoten 88

Wassergeburt 33

-, nach Sectio 76

Wide local excision 39

Wochenbett 236

Zäkumruptur 236

Zwillinge 14

-, monochorial/dichorial 64

Zwillingsschwangerschaft 64

Zytokine 113

\section{KARGER}

(C) 2007 S. Karger AG, Basel

Fax +41 613061234

E-Mail karger@karger.ch

www.karger.com 